\title{
High Energy Exoplanet Transits
}

\author{
Joe Llama ${ }^{1}$ and Evgenya L. Shkolnik ${ }^{2}$ \\ ${ }^{1}$ Lowell Observatory, 1400 W. Mars Hill Road, Flagstaff, AZ. 86001. USA \\ email: joe.llama@lowell.edu \\ ${ }^{2}$ ASU School of Earth and Space Exploration, Tempe, AZ 85287. USA \\ email: shkolnik@asu.edu
}

\begin{abstract}
X-ray and ultraviolet transits of exoplanets allow us to probe the atmospheres of these worlds. High energy transits have been shown to be deeper but also more variable than in the optical. By simulating exoplanet transits using high-energy observations of the Sun, we can test the limits of our ability to accurately measure the properties of these planets in the presence of stellar activity. We use both disk-resolved images of the Solar disk spanning soft $\mathrm{X}$-rays, the ultraviolet, and the optical and also disk-integrated Sun-as-a-star observations of the Ly $\alpha$ irradiance to simulate transits over a wide wavelength range. We find that for stars with activity levels similar to the Sun, the planet-to-star radius ratio can be overestimated by up to $50 \%$ if the planet occults an active region at high energies. We also compare our simulations to high energy transits of WASP-12b, HD 189733, 55 Cnc b, and GJ 436b.
\end{abstract}

Keywords. Sun: activity, stars: activity, stars: spots, (stars:) planetary systems

\section{Introduction}

High energy exoplanet transits offer the exciting opportunity to study the atmospheres of planets outside our Solar System. In particular, near-ultraviolet (NUV), and farultraviolet (FUV) transits probe the upper-most atmospheres of planets, where this light is absorbed. Multi-wavelength transits have been used to determine the composition of the atmosphere through transmission spectroscopy (see for example Sing et al. 2011; Gibson et al. 2012; Nikolov et al. 2014; Stevenson et al. 2014; Pont et al. 2013). Determining the parts-per-million change in light due to the atmosphere of an exoplanet requires an extremely accurate and precise measurement of the planet-to-star radius ratio $\left(R_{p} / R_{\star}\right)$. Understanding the impact stellar activity has on these measurements is, therefore, crucial since activity will alter the depth and shape of the transit profile and the resultant $\left(R_{p} / R_{\star}\right)$ measurement.

The signature of stellar activity has been seen in a many transit light curves across the entire wavelength spectrum. In the optical, bumps in the transit light curve correspond to the planet occulting a star spot on the stellar surface. By tracking the location of these spots over consecutive transits, the spin-orbit alignment of the exoplanet can be inferred and agrees well with Rossiter-Mclaughlin measurements (Winn et al. 2010; Sanchis-Ojeda \& Winn 2011). For misaligned systems, it is possible to track changes in the phase location of the spots that correspond to latitudinal changes in the star spot distribution allowing us to recover stellar butterfly patterns and differential rotation patterns (Llama et al. 2012; Sanchis-Ojeda et al. 2013; Davenport et al. 2015). These observations are providing insight into stellar dynamos (Berdyugina 2005).

In the NUV, Hubble Space Telescope observations of WASP-12b have revealed an asymmetric transit when compared to the optical data (Fossati et al. 2010; Haswell et al. 2012). The NUV light curve is deeper than the optical transit, and also begins before but ends simultaneously with the optical transit. This early ingress suggests the presence of 
additional asymmetrically-distributed occulting material in the NUV leading the orbit of the planet.

There have been a number of explanations in the literature to explain such asymmetrical transits. One theory is that very close-in planets are heavily inflated and likely to be overflowing their Roche lobes, resulting in an accretion stream from the planet onto the star (Vidal-Madjar et al. 2003; Gu et al. 2003; Ibgui et al. 2010; Lai et al. 2010). An alternative suggestion is that the NUV early-ingress is the observational signature of the stellar wind colliding with the magnetosphere of the planet, resulting in a magnetic bow shock (Vidotto et al. (2010)). From the timing difference between the early-ingress and optical transits, Vidotto et al. (2010) were able to determine the magnetosphere stand-off distance to be $\sim 5 R_{p}$. From this, they were able to constrain the magnetic field strength of WASP-12b to be $<24$ G. Llama et al. (2011) modeled this idea using a Monte-Carlo Radiative Transfer Simulation and were able to fit the HST NUV light curve with a bow shock model with parameters similar to those found by Vidotto et al. (2010).

In the FUV, Ly $\alpha$ transits have been used to study both the atmospheric escape and inflated atmospheres of close-in exoplanets. Since these planets orbit much closer to their parent star, they will be subjected to increased levels of stellar irradiance which will heat the planet's exosphere and may lead to mass loss (Lammer et al. 2003; Lecavelier des Etangs et al. 2004). Deep Ly $\alpha$ transits have been observed using HST/STIS, hinting at the presence of extended atmospheres around HD 209458b, a hot-Jupiter orbiting a G star (Vidal-Madjar et al. 2003, 2004; Ben-Jaffel \& Sona Hosseini 2010; Vidal-Madjar et al. 2004) and of HD 189733b, a hot Jupiter orbiting a more active K dwarf (Lecavelier Des Etangs et al. 2010; Lecavelier des Etangs et al. 2012; Bourrier et al. 2013).

Ehrenreich et al. (2012) used HST/STIS to observe 55 Cnc searching for the transit of $55 \mathrm{Cnc}$ b, which has yet to be observed in optical photometry. Their data showed a transit depth of $\sim 7.5 \%$, suggesting that this planet's atmosphere is grazing the stellar disk. FUV observations of the transit of GJ 436b have also revealed that this heavily irradiated hot Neptune orbiting an $M$ dwarf is undergoing mass loss with the transit exhibiting a late egress and a transit depth of $\sim 25 \%$, far greater than the $0.69 \%$ optical transit depth (Kulow et al. (2014)). A further study of this system by Ehrenreich et al. (2015) found an even deeper asymmetric Ly $\alpha$ light curve with a transit depth of $\sim 56 \%$ and a cometary tail trailing the orbit of the planet.

Multi-epoch observations of HD 189733 in Ly $\alpha$ have shown variability in the FUV transit depth of this planet. In 2010 April there was no detectable Ly $\alpha$ transit; however, a $14.4 \pm 3.6 \%$ transit was observed in 2011 September (Lecavelier des Etangs et al. 2012; Bourrier et al. 2013). The 2011 September transit depth is $\sim 10 \times$ deeper than the optical transit and has been attributed to strong atmospheric escape from the planet which is likely correlated to the stellar wind strength of the star (Bourrier et al. 2013).

X-ray observations of HD 189733b's transit have been made using Chandra by Poppenhaeger et al. (2013). They observed a total of seven transits of HD 189733b and found a combined transit depth of $6 \%-8 \%$, some $3 \times$ larger than the optical transit depth. The authors note that stellar activity is clearly visible in their data, and depending on which observations they choose to combine, the resultant transit depth varies between $2.3 \%-9.4 \%$.

In this proceedings, we review the work of Llama \& Shkolnik (2015) and Llama \& Shkolnik (2016), where high energy observations of the Sun were used as input into a transit code to simulate the impact of stellar activity on exoplanet transits. 

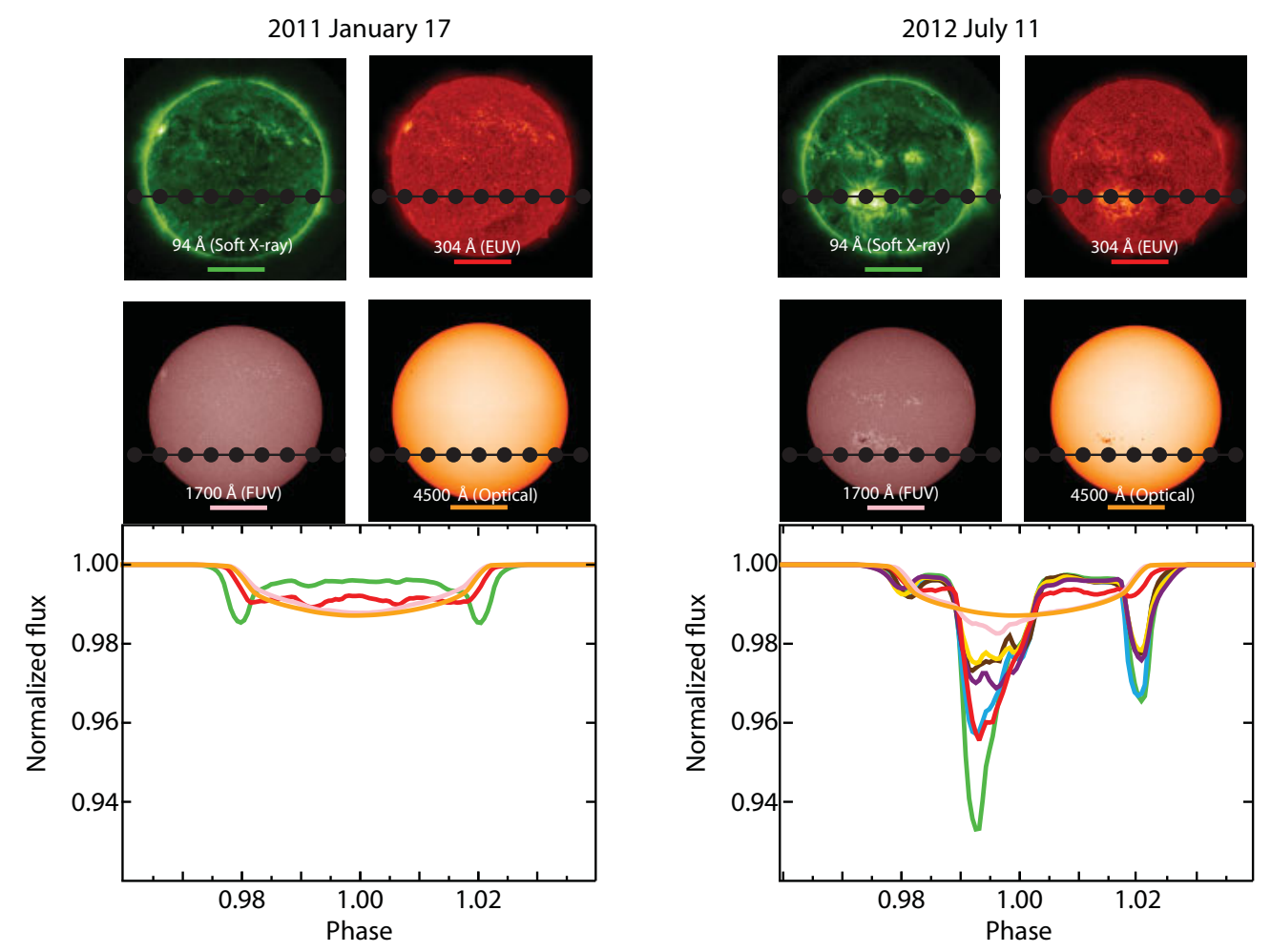

Figure 1. Top: Two sets of images of the Sun taken with NASA's SDO/AIA spanning the soft $\mathrm{X}$-ray/EUV through to the optical. The left panel was taken while the Sun was quiet, while the right panel was obtained when there were a large number of active regions on the solar disk. Overplotted is the trajectory of the simulated hot Jupiter. Bottom: The simulated exoplanet transit for each of the light curves. Figure adapted from Llama \& Shkolnik (2015).

\section{Solar Observations}

Using the Sun-as-a-star allows us to study the impact of stellar activity on exoplanet observations where we can directly observe the solar surface. NASA's Solar Dynamics Observatory (SDO) has been observing the Sun continuously since 2010. In this work, we make use of the data from the Atmospheric and Imaging Assembly (AIA) on board SDO. AIA images the solar disk in 10 wavelengths every 10 seconds, with a wavelength coverage spanning soft-Xray/EUV through to the optical. The images have a spatial resolution of 1" using a CCD of $4096 \mathrm{px}^{2}$. In Llama \& Shkolnik (2015) we obtained images of the Sun in all ten wavelengths once every 24 hours between 2011 January 01 and 2014 September 01. In total our sample was comprised of 1300 images at each of the ten wavelengths.

Figure 1 shows images of the Sun from SDO/AIA in four wavelengths spanning the soft X-ray/EUV through to the optical on two separate dates. The short wavelength (EUV) images show how active regions on the solar disk appear as bright, extended features, whereas at longer wavelengths (FUV and optical) the active regions appear as smaller, dark regions.

\section{Transit Model}

Our transit model is based on that of Llama et al. (2013). Each image of the Sun is used as the stellar background over which we simulate the transit of a hot Jupiter with 


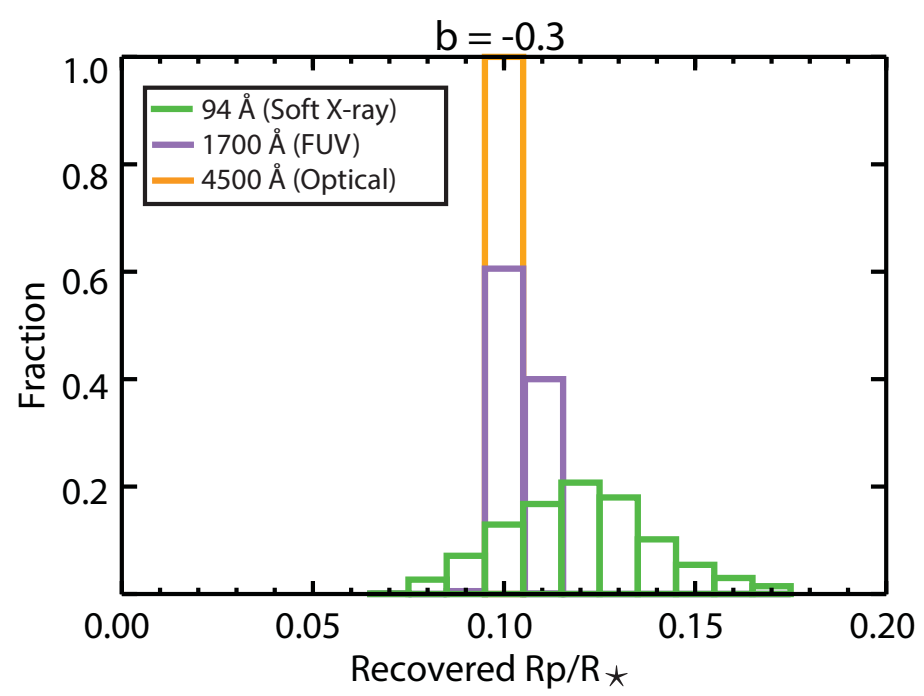

Figure 2. Histogram showing the distribution of measured $R_{p} / R_{\star}$ for the soft-Xray, FUV, and optical transits. In all cases, the input value of $R_{p} / R_{\star}=0.1$. This Figure was adapted from Llama \& Shkolnik (2015).

$R_{p} / R_{\star}=0.1$. To enable us to determine the impact of stellar activity on the simulations we assumed the planet to have the same radius in all wavelengths, i.e., the atmosphere of the planet does not change with wavelength. We also assumed the planet to be completely dark so that any region of the stellar disk occulted by the planet will have zero flux. At each step in the simulation, the position of the planet over the stellar disk is computed, and the loss in flux caused by the planet occulting that particular region of the star is summed to produce the simulated transit light curve.

\section{Transit Depths as a function of wavelength}

For each of our $\sim 1300$ images in each of the ten wavelengths, we computed the transit light curve and measured $R_{p} / R_{\star}$ using either a limb-darkened (FUV and optical) or limb-brightened (soft-Xray/EUV) transit model (see Llama \& Shkolnik (2015) for more details).

Figure 2 shows the distribution of measured $R_{p} / R_{\star}$ for all our simulated light curves. As expected, the optical histogram is single peaked and centered on the input value of $R_{p} / R_{\star}=0.1$, showing that for stars with similar activity levels as the Sun, optical star spots have little impact on our ability to accurately determine $R_{p} / R_{\star}$. At shorter wavelengths, there is a wider spread in the recovered values of $R_{p} / R_{\star}$. In the FUV, even though the active regions are dark, faculae and plage appear much brighter relative to the ambient photosphere than in the optical. Since these regions are brighter than their surroundings, the planet occults more starlight as it passes over them, resulting in a deeper transit. In the soft X-ray/EUV, active regions appear as bright extended regions, which, when occulted by the planet cause a depth increase in the transit. Since these regions are very extended relative to the optical, the recovered transit depth and hence $R_{p} / R_{\star}$, is on average, overestimated at high energies.

At short wavelengths, we find that up to $70 \%$ of the simulated transits exhibited stellar activity signatures that resulted in the measured radius of the planet to be overestimated by up to $\sim 50 \%$. In the FUV, the number of impacted transits was lower, at $\sim 20 \%$, 


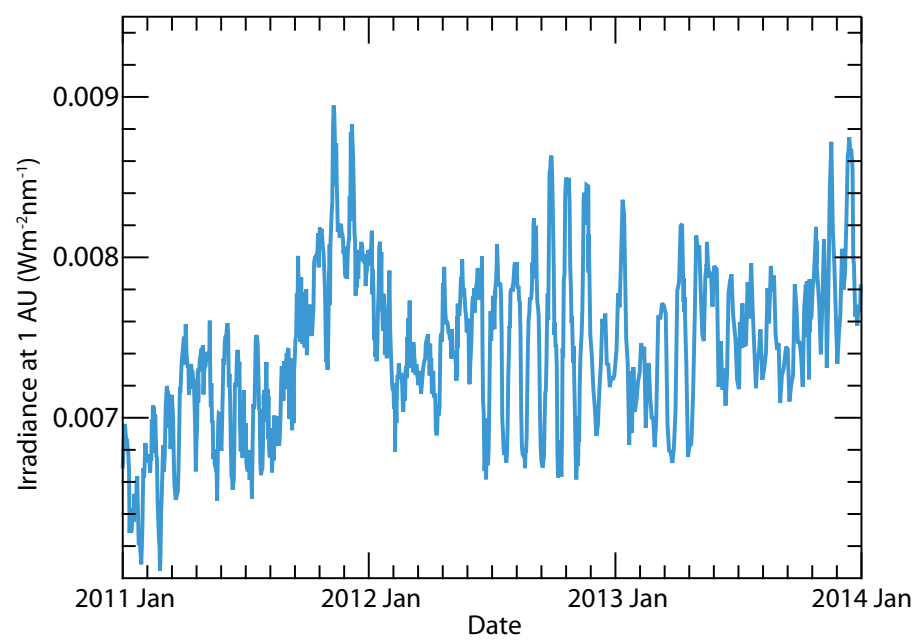

Figure 3. EVE MEGS-P Ly $\alpha$ irradiance. Figure adapted from Llama \& Shkolnik (2016).

while in the optical we find that stars with activity signatures similar to the Sun, only $5 \%$ of light curves will show localized bumps caused by the planet occulting a star spot. For a complete description of the impact of stellar activity on the light curves we refer the reader to Sections 3 and 4 of Llama \& Shkolnik (2015).

\section{5. $\operatorname{Ly} \alpha$ Transits}

In Llama \& Shkolnik (2016) we used observations of the Solar Ly $\alpha$ flux to study the impact of stellar activity of this EUV line on transit observations. The Extreme Ultraviolet Variability Experiment (EVE) instrument on board SDO measures the diskintegrated (Sun-as-a-star) EUV spectrum with a $10 \mathrm{~s}$ cadence within the wavelength range of 6.5 - $105 \mathrm{~nm}$ using the MEGS-A and MEGS-B components. Additionally, the photodiode MEGS-P monitors a $10 \mathrm{~nm}$ band centered around Ly $\alpha$ at $121.6 \mathrm{~nm}$. To minimize degradation to the instruments, MEGS-P only observes for $3 \mathrm{hr}$ a day at a 10 s cadence and once every 5 minutes every hour for the remainder of the day.

We obtained all the available EVE Level 2 line data which contain the Solar irradiance adjusted to $1 \mathrm{AU}$ at selected emission lines, including Ly $\alpha$. Figure 3 shows the Solar Ly $\alpha$ irradiance between 2011 January and 2014 January. There are two clear types of variability in this light curve. Firstly, the 27 day solar rotation period can clearly be seen. This modulation varies by up to $30 \%$; however, the timescale of the variability is less problematic for exoplanet observations which are typically taken over a few hours. The more problematic issue, however, is short term variability which hampers our ability to determine the correct normalization level and accurately measure $R_{p} / R_{\star}$.

Since the Ly $\alpha$ data is not disk resolved, we could not use the transit model of Llama et al. (2013). Instead, we isolated each $3 \mathrm{hr}$ section of continuous Ly $\alpha$ monitoring and combine it with a simulated limb-darkened transit using the code developed by Mandel \& Agol (2002) with the same $10 \mathrm{~s}$ cadence. Again, we chose the input $R_{p} / R_{\star}=0.1$, and the $3 \mathrm{hr}$ transit was comprised of the $1 \mathrm{hr}$ transit event and $1 \mathrm{hr}$ of out-of-transit data on either side of the transit for normalization. To measure $R_{p} / R_{\star}$ we performed a $\chi^{2}$ test with Mandel \& Agol (2002) light curves of varying $R_{p} / R_{\star}$ to find the best fit. 


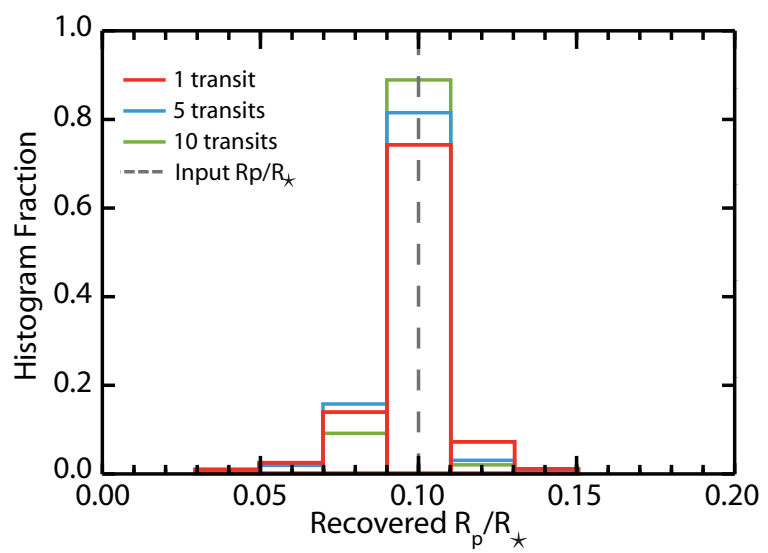

Figure 4. Histogram of recovered $R_{p} / R_{\star}$ for our Ly $\alpha$ simulations. For a single transit, the correct answer is measured $\sim 75 \%$ of the time. Once 10 consecutive transits are averaged together, the correct answer is measured over $90 \%$ of the time. Figure adapted from Llama \& Shkolnik (2016).

It is worthy of note that we used disk resolved data, as such all spatial information is lost this experiment provides the limits of probing the upper atmospheres of exoplanets at this important wavelength.

In total we simulated 1100 transits. Figure 4 shows the distribution of measured $R_{p} / R_{\star}$ for this set of simulations. The histogram for 1 transit shows that we obtain the input value of $R_{p} / R_{\star}=0.1$ (within error of $\pm 5 \%$ ) in $\sim 75 \%$ of the simulations. In the X-ray transit work of Poppenhaeger et al. (2013) they obtained seven transits and combined them in an effort to combat stellar activity. In Figure 4 we show histograms for combining 5 and 10 consecutive transits. Once 10 transits are combined, the correct answer was measured over $90 \%$ of the time. This, therefore, shows that despite stellar activity changes on the surface of the star between observations, combining multiple transits together increases the likelihood of measuring the correct value of $R_{p} / R_{\star}$.

\section{Summary}

Accounting for stellar activity on high energy exoplanet transits is imperative in ensuring the correct value of $R_{p} / R_{\star}$ is measured. The Sun offers a unique opportunity to study the impact of stellar activity by simulating transits of a hot Jupiter over high cadence data of the solar disk. Using disk-resolved images of the Sun from NASA's SDO/AIA instrument we have simulated over 13,000 transits in wavelengths spanning soft X-rays/EUV through to the FUV, and optical.

Our simulations show that in $70 \%$ of short wavelength transits the light curves show signatures of stellar activity that would result in the derived value of $R_{p} / R_{\star}$ differing from the correct value. As expected, this number decreased at longer wavelengths.

By using disk integrated Ly $\alpha$ data from NASA's SDO/EVE instrument we were able to place lower limits on the impact stellar activity has on observations at this wavelength. In none of our 1000 simulated Ly $\alpha$ transits were we able to reproduce the observed early ingress seen in the NUV light curves of WASP-12b observed by Fossati et al. (2010); Haswell et al. (2012), adding strength to the conclusion that this asymmetry is the signature of a magnetospheric bow shock (Vidotto et al. 2010; Llama et al. 2011). In none of our simulations were we able to reproduce the large transit of $55 \mathrm{Cnc}$ b observed 
by Ehrenreich et al. (2012), again strengthening the conclusion that this planet has an extended atmosphere that is grazing the stellar disk.

We conclude that it is vital to account for stellar activity in high-energy transit observations. Simultaneous observations in the optical and long baselines can help accurately determine the normalization level and disentangle planetary properties from stellar activity.

\section{References}

Ben-Jaffel, L. \& Sona Hosseini, S. 2010, ApJ, 709, 1284

Berdyugina, S. V. 2005, Living Reviews in Solar Physics, 2, 8

Bourrier, V., Lecavelier des Etangs, A., Dupuy, H., et al. 2013, A\&AA, 551, A63

Davenport, J. R. A., Hebb, L., \& Hawley, S. L. 2015, ApJ, 806, 212

Ehrenreich, D., Bourrier, V., Bonfils, X., et al. 2012, A\& A, 547, A18

Ehrenreich, D., Bourrier, V., Wheatley, P. J., et al. 2015, Nature, 522, 459

Fossati, L., Haswell, C. A., Froning, C. S., et al. 2010, ApJL, 714, L222

Haswell, C. A., Fossati, L., Ayres, T., et al. 2012, ApJ, 760, 79

Gibson, N. P., Aigrain, S., Pont, F., et al. 2012, MNRAS, 422, 753

Gu, P.-G., Lin, D. N. C., \& Bodenheimer, P. H. 2003, ApJ, 588, 509

Ibgui, L., Burrows, A., \& Spiegel, D. S. 2010, ApJ, 713, 751

Kulow, J. R., France, K., Linsky, J., \& Loyd, R. O. P. 2014, ApJ, 786, 132

Lai, D., Helling, C., \& van den Heuvel, E. P. J. 2010, ApJ, 721, 923

Lammer, H., Selsis, F., Ribas, I., et al. 2003, ApJL, 598, L121

Lecavelier des Etangs, A., Vidal-Madjar, A., McConnell, J. C., \& Hébrard, G. 2004, A\&SA, 418, L1

Lecavelier Des Etangs, A., Ehrenreich, D., Vidal-Madjar, A., et al. 2010, A\&GA, 514, A72

Lecavelier des Etangs, A., Bourrier, V., Wheatley, P. J., et al.2012, A\&̛A, 543, L4

Llama, J., Wood, K., Jardine, M., et al. 2011, MNRAS, 416, L41

Llama, J., Jardine, M., Mackay, D. H., \& Fares, R. 2012, MNRAS, 422, 72

Llama, J., Vidotto, A. A., Jardine, M., et al. 2013, MNRAS, 436, 2179

Llama, J. \& Shkolnik, E. L. 2015, ApJ, 802, 41

Llama, J. \& Shkolnik, E. L. 2016, ApJ, 817, 81

Mandel, K. \& Agol, E. 2002, ApJL, 580, L171

Nikolov, N., Sing, D. K., Pont, F., et al. 2014, MNRAS, 437, 46

Pont, F., Sing, D. K., Gibson, N. P., et al. 2013, MNRAS, 432, 2917

Poppenhaeger, K., Schmitt, J. H. M. M., \& Wolk, S. J. 2013, ApJ, 773, 62

Sanchis-Ojeda, R. \& Winn, J. N. 2011, ApJ, 743, 61

Sanchis-Ojeda, R., Winn, J. N., Marcy, G. W., et al. 2013, ApJ, 775, 54

Sing, D. K., Pont, F., Aigrain, S., et al. 2011, MNRAS, 416, 1443

Stevenson, K. B., Bean, J. L., Seifahrt, A., et al. 2014, AJ, 147, 161

Vidal-Madjar, A., Lecavelier des Etangs, A., Désert, J.-M., et al. 2003, Nature, 422, 143

Vidal-Madjar, A., Désert, J.-M., Lecavelier des Etangs, A., et al. 2004, ApJL, 604, L69

Vidotto, A. A., Jardine, M., \& Helling, C. 2010, ApJL, 722, L168

Winn, J. N., Johnson, J. A., Howard, A. W., et al. 2010, ApJL, 723, L223 